\title{
O QUE LER PARA ENTENDER A ARQUITETURA DOS TEMPLOS DÓRICOS GREGOS: UMA ORGANIZAÇÃO BIBLIOGRÁFICA SISTEMÁTICA COM INTRODUÇÃO PARA MAIS DE UM SÉCULO DE PESQUISAS
}

Claudio Walter Gomez Duarte ${ }^{1}$

\begin{abstract}
Resumo
Este artigo é a tradução e atualização do artigo "BIBLIOGRAPHIE SELECTIVE CONCERNANT LES TEMPLES GRECS DU 19e AU 21e SIECLE" publicado no periódico CAIETE ARA ARHITECTURÀ. RESTAURARE. ARHEOLOGIE, 3, 2012, em Bucareste, Romênia. Objetivamos uma organização bibliográfica com uma introdução ao tema da "concepção arquitetônica dos templos dóricos perípteros gregos". A nossa experiência de mais de uma década de pesquisa trouxe à luz uma bibliografia muito extensa e complexa de se administrar, suscitando a pergunta: O que ler sobre o assunto? Muito se escreveu sobre o tema até o presente e acreditamos que um recorte importante, para organizar essa bibliografia, se faça a partir das últimas duas décadas do século XIX. Uma estratégia de pesquisa foi a de propor uma organização bibliográfica cronológica e temática. Esta organização revelou uma estrutura e um caminho, revelou, também, que diversos tipos de abordagens devem ser associados para termos um acesso a um tema tão complexo como o da concepção arquitetônica. Apresentamos de modo introdutório: o tema da arquitetura dórica dos templos gregos, a importância da metrologia grega para este tipo de estudo e a relevância de Vitrúvio, a principal fonte histórica para a arquitetura na antiguidade.
\end{abstract}

\section{Palvras-chave}

Arquitetura grega; templos dóricos gregos; templos perípteros; design dórico; proporções e módulos.

\footnotetext{
${ }_{1}^{1}$ Pós-doutorando, Faculdade de Arquitetura e Urbanismo, CNPq, Universidade de São Paulo São Paulo, Brasil. email: rodrigo.araujo.lima@usp.br
} 
Tradicionalmente precisão, regras de proporção e rigor matemático têm sido vistos como parte essencial da sofisticada prática arquitetônica grega. É evidente que a arquitetura grega atingiu um alto grau de padronização, principalmente na maneira de produzir templos, e isso se desenvolveu de modo mais normativo dentro da ordem dórica. A realidade arqueológica mostra, contudo, que as "regras" não foram estabelecidas de maneira totalmente mecanizada, como estabeleceu Vitrúvio em seu tratado De Arquitetura, dando margem a variações na decorrência de séculos de aperfeiçoamento e variando de região para região e, também, de acordo com as exigências especificas do local, do culto e da imagem dentro da edificação. Se existem, no entanto, monumentos relativamente bem fundamentados matematicamente, supõe-se que os arquitetos tivessem boas bases teóricas. Mas, não é necessariamente o caso de todos os monumentos gregos. De fato, não é um compromisso formal da arquitetura com a matemática que observamos, pelo menos, no tratamento analítico dado aos templos dóricos pelos especialistas da escola moderna, que tenta reconstruir o percurso feito pelos arquitetos gregos ao conceber seus templos dóricos. O que temos nas interpretações mais bem fundamentadas é a constatação da aplicação consciente de proporções simples e restritas somente a alguns elementos arquitetônicos do edifício, e mesmo as interpretações modulares modernas se distanciam muito de um receituário à moda de Vitrúvio - a nossa fonte histórica mais importante sobre a arquitetura na Antiguidade greco-romana.

Muito se escreveu sobre o tema, principalmente no século XX, e hoje, no início do século XXI, nos deparamos com uma exaustiva e complexa bibliografia sobre o assunto, que pretendemos organizar e apresentar. Diversos tipos de estudos abordam a arquitetura dos templos dóricos gregos e, sem dúvida, os mais importantes são os trabalhos de escavação, reconstituição, os estudos individuas para cada templo e os manuais de arquitetura. Essa bibliografia não será apresentada nesse artigo, pois é enorme e está muito bem documentada, pelo menos para os principais templos, na quinta edição do livro clássico de G. Gruben, Griechische Tempel und Heiligtümer, München, Wissenschaftliche Buchgesellschaft, 2001, p. 491-516. Acreditamos que um estudo "completo" sobre a arquitetura dos templos dóricos gregos deva contemplar diferentes tipos de abordagens. A primeira delas, e, portanto, a fundamental, que já mencionamos, consiste nas obras de referência para cada templo. Damos especial atenção, nesse artigo, para as obras dedicadas aos estudos de templos gregos em geral, ou seja, tratando de vários templos simultaneamente, estes visam questões de desenho arquitetônico, concepção e proporcionalidade. Este item é apresentado em duas listas, 
uma só de livros e teses e a outra somente composta por artigos. Essas duas listas são fundamentais para as questões de interpretação da arquitetura dórica. Outro assunto importante da nossa organização bibliográfica é o complexo tema da metrologia grega.

Ao contrário das civilizações egípcia e romana para as quais uma abundante documentação chegou até nós, seja através de instrumentos de medida e padrões inscritos em rochas, para a civilização grega contamos com uma escassa documentação a respeito. No campo da análise da arquitetura e do urbanismo a indução de unidades de medidas a partir dos próprios edifícios não evitou resultados discrepantes e falta de consenso entre os pesquisadores. Até recentemente contávamos basicamente com o relevo metrológico de Oxford, a mesa de medidas de Assos e alguns compassos recuperados da antiguidade grega. O cenário muda significativamente com a publicação de novas descobertas: o relevo metrológico de Salamina, em 1990; uma régua e um esquadro de arquiteto em 2006. Apresentamos na lista sobre a metrologia grega os livros mais importantes e os principais artigos produzidos desde o final do século XIX. A metrologia grega é hoje assunto em voga para os estudos de arquitetura. Outro tema de suma importância para o estudo dos templos dóricos gregos é a fonte histórica Vitrúvio.

Vitrúvio (Marcus Vitruuius Pollio) nasceu provavelmente entre 80/70 a.C., cresceu e educou-se na Campânia ou Roma. Seu tratado, De Arquitetura, foi provavelmente escrito e publicado em 30/20 a.C. e é considerado a mais importante fonte que chegou até nós sobre arquitetura antiga - fortemente influenciado por fontes gregas creditadas pelo próprio autor. De Arquitetura aborda as questões práticas de modo auto-explicativo e a sua própria formação, arquiteto-engenheiro militar, pode ter influenciado a maneira de lidar com as abstrações, muito embora a teoria tenha ocupado um lugar importante em seu programa como um todo. O livro IV do tratado de Vitrúvio traz um relato importante sobre como projetar templos de ordem dórica. Pela sua importância, o tema "Vitrúvio" é apresentado em nossa organização bibliográfica em três listas diferentes. A primeira lista relaciona as mais importantes traduções da obra De Arquitetura, feitas nos séculos XX e XXI. Esta relação traz traduções diretas do latim, edições bibingues e traduções comentadas. A segunda lista relaciona um conjunto de livros sobre os estudos vitruvianos, realizados para a arquitetura grega e os templos. A terceira lista separadamente relaciona artigos sobre o mesmo tema.

Acreditamos que a convergência desses materiais bibliográficos (os estudos individuais de cada templo, os manuais de arquitetura grega, os estudos especiais sobre a concepção dos templos gregos, os estudos sobre 
a metrologia grega, o tratado de Vitrúvio e os estudos vitruvianos) forme um corpus bibliográfico consistente para os estudos sobre a concepção arquitetônica dos templos dóricos gregos. Optamos pela apresentação cronológica da bibliografia, em vez da apresentação tradicional, pois, acreditamos que assim traçamos um panorama de como os estudos foram se realizando com o passar do tempo, e fica mais claro perceber em que momentos temos maior quantidade de estudos acumulados. Usamos as datas entre colchetes para designar a publicação mais recente do mesmo livro. A seguir apresentamos a organização bibliográfica.

1. Obras de Referência sobre a concepção dos templos dóricos gregos

2. Artigos sobre a concepção dos templos dóricos gregos.

3. Metrologia grega.

4. Vitrúvio, De Arquitetura: traduções, traduções bilingue e traduções comentadas.

5. Vitrúvio: livros sobre estudos vitruvianos para os templos gregos.

6. Vitrúvio: artigos sobre estudos vitruvianos para os templos gregos.

Abreviaturas: seguimos em primeiro lugar, L'ANNÉE PHILOLOGIQUE ONLINE e em segundo lugar AJA ABBREVIATIONS.

AA: Archäologischer Anzeiger. Berlin, de Gruyter.

AantHung: Acta Antiqua Academiae Scientiarum Hungaricae. Budapest, Akadémiai Kiadó.

AJA: American Journal of Archaeology. New York, Archaeol. Inst. of America.

AZ: Archäologische Zeitung. [fonte: AJA 95, p. 7, 1991]

BA: Bollettino d'Arte del Ministero per i beni culturali e ambientali. Roma, Ist. Poligraf. E Zecca dello Stato.

BABesch: Bulletin Antieke Beschaving. Leiden, Rapenburg 26.

BSA: Annual of the British School at Athens. London, Univ. of London, Institute of Classical Studies.

Dacia: Revue d'archéologie et d'histoire ancienne $=$ journal of archaeology and ancient history. Bucarest : Éd. de l'Académie roumaine.

Gnomon: Kritische Zeitschrift für die gesamte klassische Altertumswissenschaft. München, Beck. 
Hephaistos: New approaches to classical archaeology and related fields : Kritische Zeitschrift $\mathrm{zu}$ Theorie und Praxis der Archäologie und angrenzender Gebiete, Sonderband.

JDAI: Jahrbuch des Deutschen Archäologischen Instituts. Berlin, de Gruyter.

JHS: Journal of Hellenic Studies. London, Soc. for the Promotion of Hellenic Studies.

MarbWPr: Marburger Winckelmann-Programm.

MDAI(A): Mitteilungen des Deutschen Archäologischen Instituts (Athen. Abt.). Berlin, Mann.

MDAI(I): Mitteilungen des Deutschen Archäologischen Instituts (Abt. Istambul). Tübingen, Wasmuth.

$\operatorname{MDAI}(\mathrm{R})$ : Mitteilungen des Deutschen Archäologischen Instituts (Röm. Abt.) Mainz, von Zabern.

Oath: Opuscula Atheniensia: acta Inst. Athen. Regni Sueciae. Lund, Åström.

ÖJH: Jareshefte des Österreichischen Archäologischen Institutes in Wien.

RendLinc: Rendiconti dell'Accademia nazionale dei Lincei, Classe di scienze morali, storiche e filologiche.

\section{OBRAS DE REFERÊNCIA SOBRE A CONCEPÇÃO DOS TEMPLOS DÓRICOS GREGOS}

1888. PENROSE, F. C. An investigation of the principles of Athenian architecture; or, The results of a recent survey conducted chiefly with reference to the optical refinements exhibited in the construction of the ancient buildings at Athens. $2^{\mathrm{a}}$, London, Macmillan.

1892. IVANOFF, S. A. Architektonische Studien, I, Aus Griechenland. Berlin, Deutsches Archäologisches Institut.

1899. KOLDEWEY, R.; PUCHSTEIN, O. Die Griechischen Tempel in Unteritalien und Sicilien. Berlin, Asher.

1912. GOODYEAR, W. H. Greek Refinements. Studies in temperamental architecture. New Haven, Yale University Press. 
1918. THEUER, M. Der griechisch-dorische peripteraltempel; ein beitrag zur antiken proportionslehre. Berlin, Ernst Wasmuth.

1929. WEICKERT, C. Typen der archaischen Architektur in Griechenland und Kleinasien. Augsburg, DR. Benno filser.

1935. RIEMANN, H. Zum griechischen Peripteraltempel; seine Planidee und ihre Entwicklung bis zum Ende des 5. Jhds. Tese de doutorado, Düren, Spezial-Dissertations-Buchdr.

1958. RIEMANN, H. Der antike Tempel. Darmstadt, Wissenschaftlichen Buchgesellschaft.

1941. KRAUSS, F. Paestum - die griechischen Tempel. Berlin, Mann. [5 ed., 1984].

1941. RODENWALDT, G. Griechische Tempel. Berlin, Deutscher Kunstverlag. [2a $\left.{ }^{\mathrm{a}}, 1951\right]$.

1950. DINSMOOR, W. B. The architecture of ancient Greece: an account of its historic development. $3^{\mathrm{a}}$ ed., London, B.T. Batsford.

1950. RAUBITSCHEK, I. K. Ionicizing-Doric architecture: a stylistic study of Greek Doric architecture of the sixth and fifth centuries B.C. Tese de doutorado, New York, Columbia University.

1951. KOCH, H. Der griechisch-dorische Tempel. Stuttgart, J.B. Metzler.

1957. BUNDGAARD, J. A. Mnesicles, a Greek architect at work. København, Gyldendal.

1957. JEPPESEN, K. Paradeigmata; three mid-fourth century main works of Hellenic architecture, reconsidered. Aarhus, Aarhus University Press.

1959. GERKAN, A. Von. Von antiker Architektur und Topographie; gesammelte Aufsatze. Stuttgart, Kohlhammer.

1961. BERVE, H; GRUBEN, G. Griechische Tempel und Heiligtümer. München, Hirmer Verlag. [5a ed., 2001].

1964. KÄHLER, H. Der griechische Tempel: Wesen und Gestalt. Berlin, Mann. [2a, 1981].

1968. PETRONOTIS, A. Bauritzlinien und andere Aufschnürungen am Unterbau griechischer Bauwerke in der Archaik und Klassik: eine Studie zur 
Baukunst und -technik der Hellenen. Tese de doutorado, München, Techn. Hochsch.

1973. PETRONOTIS, A. Zum Problem der Bauzeichnungen bei den Griechen, Athens, Dodona Verlag.

1969. DRERUP, H. Griechische Baukunst in geometrischer Zeit. Gottingen, Vandenhoeck \& Ruprecht.

1970. RICHARD, H. Vom Ursprung des dorischen Tempels. Bonn, R. Habelt.

1973. EITELJORG, H. The Greek architect of the fourth century B.C.: Master craftsman or master planner. Tese de doutorado, Ann Arbor, Mich.: Univ. Microfilms Internat.

1976. KALPAXIS, A. E. Fruharchaische Baukunst in Griechenland und Kleinasien. Tese de doutrado, Athen, Universität Heidelberg.

1977. COULTON, J. J. Ancient Greek architects at work: problems of structure and design. Ithaca, New York, Cornell University Press.

1979. KNELL, H. Perikleische Baukunst. Darmstadt, Wissenschaftliche Buchgesellschaft.

1980. KNELL, H. Grundzüge der griechischen Architektur, Darmstadt, Wissenschaftliche Buchgesellschaft. [2 ${ }^{\mathrm{a}}$ ed. 1988].

1984. HOEPFNER, W. (ed.) Bauplanung und Bautheorie der Antike: Bericht uber ein Kolloquium veranstaltet vom Architekturreferat des Deutschen Archäologischen Institutes <DAI> mit Unterstutzung der Stiftung Volkswagenwerk in Berlin vom 16.11. bis 18.11.1983. Berlin, Wasmuth.

1984. BERGER, E. (ed.) Parthenon-Kongress Basel: Referate und Berichte, 4. bis 8. April 1982. Mainz, P. von Zabern.

1984. MERTENS, D. Der Tempel von Segesta: und die dorische Tempelbaukunst des griechischen Westens in klassischer Zeit. Mainz, P. von Zabern.

1993. MERTENS, D. Der alte Heratempel in Paestum: und die archaische Baukunst in Unteritalien. Mainz, P. von Zabern.

2006. MERTENS, D. Stälte und Bauten der Westgriechen: von der Kolonisationszeit bis zur Krise am Ende des 5. Jh. v. Chr. München, Hirmer. 
1985. Le dessin d'architecture dans les sociétés antiques: actes du Colloque de Strasbourg, 26-28 janvier 1984. Travaux du Centre du Recherche sur le Proche Orient et la Grèce Antique 8. Leiden, E. J. Brill.

1985. HOWE, T. N. The invention of the Doric order. Tese de doutorado, Cambridge, Harvard Universty.

1985. WEICKENMEIER, N. Theorienbildung zur Genese des Triglyphon: Versuch einer kritischen Bestandsaufnahme. Tese de doutorado, Darmstadt, Technische Universität Darmstadt.

1986. SHIMMIN, J. A. Doric Temple proportions: a statistical solution to a problem in classical archaeology. Colorado, [s. ed.].

1988. BÜSING, H. H.; HILLER, F. (eds.) Bathron: Beiträge zur Architektur und verwandten Künsten: für Heinrich Drerup zu seinem 80. Geburtstag von seinen Schuilern und Freunden. Saarbücken, Saarbrucker Druckerei und Verlag.

1988. MÜLLER-WIENER, W. Griechisches Bauwesen in der Antike. München, Beck.

1991. CLARKE, D. A. Doric proportions in Greek monuments: 600-110 B.C. Tesis de doutorado, Toronto, University of Toronto.

1991. KLEIN, N. L. The origin of the Doric order on the mainland of Greece: form and function of the geison in the Archaic period. Tese de doutorado, Ann Arbor, Mich. Bryn Mawr College.

1992. SCHMITT, R. Handbuch zu den Tempeln der Griechen. Frankfurt am Main, Peter Lang.

1993. BANKEL, H. Der späarchaische Tempel der Aphaia auf Aegina. Berlin, Walter de Gruyter .

1993. HEISEL, J. P. Antike Bauzeichnungen. Darmstadt, Wissenschaftliche Buchgesellschaft.

1993. C. Höker Planung und Konzeption der klassischen Ringhallentempel von Agrigent: Uberlegungen zur Rekonstruktion von Bauentwürfen des 5. Jhs. v. Chr. Frankfurt, Peter Lang.

1994. LEWIS, D. C. Revealing the Parthenon's logos optikos: a historical, optical and perceptual investigation of twelve classical adjustments of form, position, and proportion. Tese de doutorado, Ann Arbor, Georgia Institute of Technology. 
1994. ROCCO, G. Guida alla lettura degli ordini architettinici antichi. 1. Il dórico. Napoli, Liguori editore.

1995. KLEBINDER G. V. Der dorische Peripteraltempel in der spätarchaischen und frühklassischen Zeit. Tese de doutorado, Salzburg, Universität Salzburg.

1997. MAZARAKIS AINIAN, A. From rulers' dwellings to temples: architecture, religion and society in early Iron Age Greece (1100-700 B.C.). Jonsered, Paul Aiströms förlag.

1999. HASELBERGER, L. (ed.) Appearance and essence: refinements of classical architecture - curvature: proceedings of the Second Williams Symposium on Classical Architecture held at the University of Pennsylvania, Philadelphia, April 2-4, 1993. Philadelphia, University of Pennsylvania.

2001. BARLETTA, B. A. The origins of the Greek architectural orders. Cambridge, Cambridge University Press. [2 ${ }^{\mathrm{a}} 2009$ ].

2001. DE WAELE, J. A. K. E. et al. Il tempio dorico del Foro Triangolare di Pompei. Roma, "L'Erma" di Bretschneider.

2002. HELLMANN, M.-C. L'architecture grecque. 1: Les principes de la construction. Paris, Picard.

2006. HELLMANN, M.-C. L'architecture grecque. 2: Architecture religieuse et funéraire. Paris, Picard.

2006. MĂRGINEANU CÂRSTOIU, M. Architecture grecque et romaine. Membra disiecta. Histria XII. Bucureşti, Editura Academiei Române.

2010. DUARTE, C. W. G. Geometria e aritmética na concepção dos templos dóricos gregos. Dissertação de mestrado, São Paulo, Universidade de São Paulo.

2015. DUARTE, C. W. G. "ELEGÂNCIA" e "SUTILEZA" na concepção dos templos dóricos gregos (Sécs. V-II a.C.). Tese de doutorado, São Paulo, Universidade de São Paulo.

2011. SENSENEY, J. R. The art of building in the classical world: vision, craftsmanship, and linear perspective in Greek and Roman architecture. Cambridge, Cambridge University Press. 
2012. WOODWARD, R. J. An Architectural Investigation into the Relationship between Doric Temple Architecture and Identity in the Archaic and Classical Periods. Tese de doutorado, Sheffield, University of Sheffield.

2013. PAKKANEN, J. Classical Greek Architectural Desing: A Quantitative Approach. Helsinki: Foundation of the Finnish Institute at Athens.

2014. WILSON JONES, M. Origins of classical architecture: temples, orders and gifts to the gods in ancient Greece. New Haven: Yale University Press.

\section{ARTIGOS SOBRE A CONCEPÇÃO DOS TEMPLOS DÓRICOS GREGOS}

1882. DÖRPFELD, W. Die Proportionen und Fussmaasse: Griechischer Tempel. AZ, 39, p. 261-270.

1923. DINSMOOR, W. B. How the Parthenon Was Planned. Architecture: The Professional Architectural Monthly (Australia), part I: p. 177-180; part II: p. 241-244.

1937. GERKAN, A. Von. Review: [untitled]: RIEMANN, H. Zum griechischen Peripteraltempel - Seine Planidee und ihre. Entwicklung bis zum Ende des 5. Jhds. Duren, Rhld.: Spezial-Dissertations-Buchdruckerei, 1935. Gnomon, 13, p. 84-90.

1941. SCHLIKKER, F. W. Schaubildentwurf in griechischen Tempelbau. AA, p. 748-765.

1951. RIEMANN, H. Hauptphasen in der Plangestaltung des dorischen Peripteraltempels. In: MYLONAS, G. E. (ed.) Studies Presented to David M. Robinson. Saint Louis, Missouri, Washington University Press, p. 295-308.

1964. RIEMANN, H. Die Planung des ältesten sizilischen Ringhallentempels. MDAI(R), 71, p. 19-59.

1962. DRERUP, H. Zur Entstehung der Griechischen Tempelringhalle. In: HIMMELMANN-WILDSCHÜTZ, Von N.; BIESANTZ, H. (Hrsg.) Festschrift für F. Matz. Mainz, Rhein, P. von Zabern p. 32-38.

1965. THEODORESCU, D. Trois étapes dans l'evolution du chapiteau dorique à Histria. Dacia, 9, p. 147-162. 
1973. KNELL, H. Vier attische Tempel klassischer Zeit zum Problem der Baumeisterzeichnungen. AA, p. 94-114.

1974. KNELL, H. Bemerkungen zum dorischen Ringhallentempel mit gedrungenem Grundriß. AA, Mitteilungen des Deutschen Archäologenverbandes, 5.2, p. 49-50.

1975. KNELL, H. Dorische Peripteraltempel mit gedrungenem Grundriss. AA, p. 10-13.

1983. KNELL, H. Dorische Ringhallentempel in spät- und nachklassischer Zeit. JDI, 98, p. 203-233.

1973. WURSTER, W. W. Dorische Peripteraltempel mit gedrungenem Grundriss. AA, p. 200-211.

1974. COULTON, J. J. Towards Understanding Doric Design: The Stylobate and Intercolumniations. BSA, 69, p. 61-86.

1975. COULTON, J. J. Towards Understanding Greek Temple Design: General Considerations. BSA, 70, p. 59-99.

1979. COULTON, J. J. Doric Capitals: A Proportional Analysis. BSA, 74, p. 81-153.

1983. COULTON, J. J. Greek architects and the transmission of design. In: Architecture et société: de l'archaïsme grec à la république romaine, colloquium, Rome, 2-4 decembre 1980. CÉFR 66. Rome, École française de Rome, p. 453-470.

1985. COULTON, J. J. Incomplete preliminary planning in Greek architecture. Some new evidence. In: Le dessin d'architecture dans les sociétés antiques: actes du Colloque de Strasbourg, 26-28 janvier 1984. Travaux du Centre du Recherche sur le Proche Orient et la Grèce Antique 8. Leiden, E. J. Brill, p. 103-121.

1976. WINTER, F. E. Tradition and Innovation in Doric Design I: Western Greek Temples. AJA, 80, p.139-145.

1978. WINTER, F. E. Tradition and Innovation in Doric Design II: Archaic and Classical Doric East of the Adriatic. AJA, 82, p. 151-161.

1980. WINTER, F. E. Tradition and Innovation in Doric Design III: The Work of Iktinos. AJA, 84, p. 399-416. 
1982. WINTER, F. E. Tradition and Innovation in Doric Design IV: The Four Century. AJA, 86, p. 387-400.

1979. FALUS, R.; MEZÖS, T. Scales and Proportions on Doric Buildings. Acta Historiae Artium Academiae Scientiarum Hungaricae, 25, p. 281318.

1979. KOENIGS, W. Zum Entwurf dorischer Hallen. MDAI(I), 29, p. 209234.

1990. KOENIGS, W. Maße und Proportionen in der griechischen Baukunst.,In: BECK, H.; BOL, P. C.; BUCKLING, M. (eds.) Polyklet: Der Bildhauer der griechischen Klassik. Frankfurt, Philipp von Zabern, p. 121134.

1996. KOENIGS, W.; PHILIPP, H. Proportion und Grösse. In: SCHWANDNER, E.-L. (ed.) Säule und Gebälk: zu Struktur und Wandlungsprozess griechisch-romischer Architektur, Diskussionen zur archäologischen Bauforschung 6. Mainz am Rhein, P. von Zabern. p. 133147.

1980. DE WAELE, J.A.K.E. Der Entwurf der dorischen Templel von Akragas. AA, p.180-241.

1980. DE WAELE, J.A.K.E. Der Entwurf der dorischen Tempel von Paestum. AA, p. 367-400.

1985. DE WAELE, J.A.K.E. Le dessin d'architecture du temple grec au début de l'époque classique. In: Le dessin d'architecture dans les sociétés antiques: actes du Colloque de Strasbourg, 26-28 janvier 1984. Travaux du Centre du Recherche sur le Proche Orient et la Grèce Antique 8. Leiden, E. J. Brill, p. 87-102.

1988 (1989-1990). DE WAELE, J.A.K.E. Reflections on the Design in Classical Greek Architecture. In: Praktika XII tou Diethnous Synedriou klasikis archaiologias, 4 . Athènes, p. 205-210.

1990. DE WAELE, J.A.K.E. Review: [untitled]: MERTENS, D. Der Tempel von Segesta und die dorische Tempelbaukunst des griechischen Westens in klassischer Zeit, Mainz am Rhein, P. von Zabern, 1984. Gnomon, 62, p. 248264.

1992. DE WAELE, J.A.K.E. I grandi temple. In: BRACCESI, L.; DE MIRO, E. (eds.) Agrigento e la Sicilia greca. Atti della settimana di studio, Agrigento, 2-8 maggio 1988. Roma, "L'Erma" di Bretschneider, p. 157-205. 
1993. DE WAELE, J.A.K.E. De klassieke Griekse temple. In: Sonderdruck Bouwkunst. Studies in vriendschap voor K. Peeters, Amsterdam, p. 580-595.

1994. DE WAELE, J.A.K.E. Meten is weten. De analyse van antieke architectuur. Lampas. Tijdschrift voor Nederlandse classici, 27, p. 150160.

1996. DE WAELE, J.A.K.E. Review: [untitled]: HÖCKER, C. Planung und Konzeption der klassischen Ringhallentempel von Agrigent: Uberlegungen zur Rekonstruktion von Bauentwurfen des 5. Jhs. v. Chr. Frankfurt am Main, Peter Lang, 1993. Gnomon, 68, p. 245-252.

1980. HASELBERGER, L. Werkzeichungen am Jüngeren Didymaion. MDAI(I), 30, p. 192-215.

1983. HASELBERGER, L. Bericht über die Arbeit am Jüngeren Apollonstempel von Didyma - Zwischenbericht. MDAI(I), 33, p. 90-123.

1981. MERTENS, D. Entgegnung zu den Entwurfshypothesen von J. de Waele, AA 1980. AA, p. 426-430.

1984. MERTENS, D. Zum klassischen Tempelentwurf. In: HOEPFNER, W. (ed.) Bauplanung und Bautheorie der Antike: Bericht uiber ein Kolloquium veranstaltet vom Architekturreferat des Deutschen Archäologischen Institutes <DAI> mit Unterstutzung der Stiftung Volkswagenwerk in Berlin vom 16.11. bis 18.11.1983. Berlin, Wasmuth, p. 137-145.

1981. TOBIN, R. The Doric Groundplan. AJA, 85, p. 379-427.

1983.CERETTO CASTIGLIANO, I.; SAVIO, C. Considerazioni sulla metrologia e sulla genesi del tempio di Giunone ad Agrigento. BA, 68, p. $35-48$.

1984. BANKEL, H. Moduli an den Tempeln von Tegea und Stratos? In: HOEPFNER, W. (ed.) Bauplanung und Bautheorie der Antike: Bericht uiber ein Kolloquium veranstaltet vom Architekturreferat des Deutschen Archäologischen Institutes <DAI> mit Unterstutzung der Stiftung Volkswagenwerk in Berlin vom 16.11. bis 18.11.1983. Berlin, Wasmuth, p. 159-166.

1984. BANKEL, H. Moduli an den Tempeln von Tegea und Stratos? AA, p. $413-430$.

1985. HORIUCHI, K. Horiuchi Formulae for Analyzing Axial Intercolumniations and Stylobate Lengths - Studies on design procedure 
of doric temples (1). Journal of architecture, planning and environmental engineering transaction of AIJ, 349, p. 101-112.

1986. HÖCKER, C. Die klassischen Ringhallentempel von Agrigent: Überlegungen zu Bauplanung and Arbeitsorganisation bei der Errichtung dorischer Tempel im Bauwesen Westgriechenlands im 5. Jhs. v. Chr. Hephaistos, 8, p. 233-247.

1996. HÖCKER, C. Architektur als Metapher, Überlegungen zur Bedeutung des dorischen Ringhallentempels. Hephaistos, 14, p. 45-79.

1987. BÜSING, H. H. Eckkontraktion und Ensemble-Planung. MarbWPr, p. 14-46.

1989. GOSHIMA, R. The Determination Method of the Stylobate Proportion and the Number of Columns by using the Grid System derived the Double-Square Diagram - A Study of Design technique of Doric Peripteral Temples. Journal of Architecture, Planning and Environmental Engineering Academic Journal, 25, p. 171-181.

1992. ØSTBY, E. Dorische Tempel, Pherai. Oath, 19, p. 85-113.

1994. PAKKANEN, J. Accuracy and Proportional Rules in Greek Doric Temples. Oath. 20, p. 144-156.

1994 (1995). MĂRGINEANU CÂRSTOIU, M. Ein neuer Vorschlag für die statistische Analyse der Komposition der dorischen Kapitelle. Dacia, 3839, p. 55-108.

1996. SHIOI, K. Statistical Analysis of the Proportions Defining Façades of Doric Temples: The meaning of the "Arkhitekton" Part (1). Journal of Architecture, Planning and Environmental Engineering Academic Journal, 481, p. 187-194.

2001. WILSON JONES, M. Doric Measure and Architectural Design 2: A Modular Reading of the Classical Temple. AJA, 105, p. 675-713.

2006. WILSON JONES, M. Ancient Architecture and Mathematics: Methodology and the Doric Temple. In: DUVERNOY, S.; PEDEMONTE, O. (eds.) Nexus VI: Architecture and Mathematics. Turin, Kim Williams Books, p. 1-20.

2002. WADDELL, G. The Principal Design Methods for Greek Doric Temples and their Modification for the Parthenon. Architectural History: Journal of the Society of Architectural Historians of Great Britain, 45, p. 131. 
2005 (2006). OSTHUES, E.-W. Studien zum dorischen Eckkonflikt. JDAI, 120, p. $1-154$.

2009. SONNTAGBAUER, W. Zur Genese des klassischen Tempelentwurfes - Zu den Grundrissen der Tavole Palatine in Metapont, des Athenatempels in Paestum, des Aphaiatempels und des Älteren Poseidon-tempels in Sunion. In: EINICKE, R. et al. (ed.) Zurück zum Gegenstand - Festschrift für Andreas Furtwängler. Langenweissbach, Beier \& Beran, p. 37-49.

\section{METROLOGIA GREGA}

1882. DÖRPFELD, W. Beiträge zur antiken Metrologie. $\operatorname{MDAI}(A), 7$, p. 277-312.

1883. DÖRPFELD, W. Beiträge zur antiken Metrologie. II. Die ägyptischen Längenmaasse. MDAI(A), 8, p. 36-56.

1883. DÖRPFELD, W. Beiträge zur antiken Metrologie. III. Die königliche Elle des Herodot und der philetaerische Fuss. MDAI(A), 8, p. 342-358.

1884. DÖRPFELD, W. Erwiederung auf Fr. Hultsch "Zur Abwehr" gegen die metrologischen Beiträge. MDAI(A), 9, p. 198-202.

1885. DÖRPFELD, W. Metrologische Beiträge. IV. Das italische MaassSystem. MDAI(A), 10, p. 289-312.

1890. DÖRPFELD, W. Metrologische Beiträge. V. Das äginäisch-attische Maass-System. MDAI(A), 15, p. 167-177.

1890. DÖRPFELD, W. Metrologische Beiträge. VI. Das griechische Stadion. MDAI(A), 15, p. 177-187.

1882. HULTSCH, F. O. Griechische und römische metrologie. Graz, Weidmannsche Buchhandlung.

1884. HULTSCH, F. O. Zur Abwehr gegen W. Dörpfelds "Beiträge zur antiken Metrologie". MDAI(A), 9, p. 140-155.

1883. LEPSIUS, R. "Die ägyptischen Langenmaasse" von Dörpfeld. MDAI(A), 8, p. 227-245.

1883. MICHAELIS, A. The Metrological Relief at Oxford. JHS, 4, p. 335350. 
1934. JOHNSTON, J. Solon's Reform of Weights and Measures. JHS, 54, p. 180-184.

1940. GERKAN, A. Von. Der Tempel von Didyma und sein antikes Baumaß. ÖJH, 32, p 127-150.

1953. BERRIMAN, A. E. Historical Metrology. London; New York, Greenwood Press.

1961. DINSMOOR, W. B. The Basis of Greek Temple Design: Asia Minor, Greece, Italy. In: Atti del Settimo Congresso Internazionale di Archeologia Classica, Vol 1. Roma, p. 355-368.

1966. LORENZEN, E. Technological Studies in Ancient Metrology. København, Busck.

1971. BRONEER, O. The Foot Measure. In: BRONEER, O. Isthmia 1: The Temple of Poseidon. New Jersey, American School of Classical Studies at Athens, Appendix I.

1972. GRUBEN, G. Naxos und Paros. Dritter Vorläufiger Bericht über die Forschungskampagnen 1970 und 1971. AA, p. 319-379.

1975 (1976). WESENBERG, B. Zum metrologischen Relief in Oxford. MarbWPr, 15-22.

1984. WESENBERG, B. Der Fuss des Kallikrates. AA, p. 547-554.

1995. WESENBERG, B. Die Metrologie der griechischen Architektur: Probleme interdisziplinärer Forschung. In: AHRENS, D.; ROTTLÄNDER, R.C.A. (Hrsg.) Ordo et mensura: Internatinaler interdisziplinärer Kongress für historische metrologie 3, 1993. St. Katharinen : Scripta Mercaturae. p. 199-222.

2001 (2002). WESENBERG, B. Vitruv und Leonardo in Salamis. 'Vitruvs Proportionsfigur' und die metrologischen Reliefs. JDAI, 116, p. 357-380.

1982. BÜSING, H. H. Metrologische Beiträge. JDAI, 97, p. 1-45.

1983. BANKEL, H. Zum fußma $\beta$ attischer bauten des 5. jarhunderts v. Chr. MDAI(A), 98, p. 65-99.

1991. BANKEL, H. Acropolis-Fussmasse. AA, p. 151-163.

1984. KNELL, H. Vitruvs Metrologisches System. In: HOEPFNER, W. (ed.) Bauplanung und Bautheorie der Antike: Bericht uber ein Kolloquium 
veranstaltet vom Architekturreferat des Deutschen Archäologischen Institutes <DAI> mit Unterstutzung der Stiftung Volkswagenwerk in Berlin vom 16.11. bis 18.11.1983. Berlin, Wasmuth, p. 33-38.

1990. DEKOULAKOU-SIDERIS, I. A Metrologial Relief from Salamis. AJA, 94, p. 445-451.

1991 (1992). ROTTLÄNDER, R.C.A. Eine neu aufgendene antike Maßeinheit auf dem metrologischen Relief von Salamis. ÖJH, 61, p. 63-68.

1994. ROTTLÄNDER, R.C.A. Das neue Bild der antiken Metrologie. Alte Vorteile - Neue Beweise. ÖJH, 63, p. 1-16.

2000. WILSON JONES, M. Doric Measure and Architectural Design 1: The Evidence of the Relief from Salamis. AJA, 104, p. 73-93.

2004. PAKKANEN, J. The Toumba Building at Lefkandi: A Statistical Method for Detecting a Design-Unit. BSA, 99, p. 257-271.

2006. STIEGLITZ, R. R. Classical Greek Measures and the Builder's Instruments from the Ma'agan Mikhael Shipwreck. AJA, 110, p. 195-203.

\section{VITRÚVIO, DE ARQUITETURA: TRADUÇÕES, TRADUÇÕES BILINGUE E TRADUÇÕES COMENTADAS}

1909. CHOISY, A. Vitruve. Texte et Traduction, I-II. Paris, Lahure.

1912. PRETZEL, J. Marcus Vitruvius Pollio. Zehn Bücher über Architektur. Strassburg, Heitz (Heitz \& Mündel).

1914. MORGAN, M. H. Vitruvius. The ten books on architecture. Cambridge, Dover Publications.

1931. GRANGER, F. Vitruvius. On architecture, Books 1-5. London, New York, Loeb classical library.

1934. GRANGER, F. Vitruvius. On architecture, Books 6-10. London; New York, Loeb classical library.

1960. FERRI, S. Vitruvio. Architettura (dai libri I-VII), Roma, Fratelli Palombi.

1964. FENSTERBUSCH, C. Vitruv. Zehn Bücher uber Architektur. Berlin, Wissenschaftliche Buchgesellschaft. 
1990. GROS, P. Vitruve. De l'architecture, livre III. Paris, Les Belles Lettres. 1992. GROS P. Vitruve. De l'architecture, livre IV. Paris, Les Belles Lettres.

2015. GROS, P. et. al. Vitruve. De l'architecture. Paris, Les Belles Lettres.

1997. CORSO, A. ; ROMANO, E.; GROS, P. Vitruvio, De architectura. Torino, Giulio Einaudi Editore.

1999. ROWLAND, I. D.; HOWE, T. N. Vitruvius. Ten books on architecture. Cambridge, Cambridge University Press.

2001. SMITH, T. G. Vitruvius on architecture. New York, The Monacelli Press.

2006. MACIEL, M. J.; HOWE, T. N. Vitrúvio. Tratado de arquitectura, Lisboa, IST Press.

2009. Richard V Schofield; Robert Tavernor Vitruvius On architecture, New York, Penguin Books.

\section{VITRÚVIO: LIVROS SOBRE ESTUDOS VITRUVIANOS PARA OS TEMPLOS GREGOS}

1909. CHOISY, A. Vitruve. Analise et Figures, III-IV. Paris, Lahure.

1914. BIRNBAUM, A. Vitruvius und die griechische architektur. Wien, Kommission bei Alfred Hölder.

1940. SCHLIKKER, F. W. Hellenistische Vorstellungen von der Schö̈heit des Bauwerks nach Vitruv. Berlin, Archäologisches Institut des Deutschen Reiches.

1945. MOE, C. J. Numeri di Vitruvio. Milano, Edizioni del Milione.

1951. KOCH, H. Vom Nachleben des Vitruvs. Baden-Baden, Verlag fur Kunst und Wissenschaft.

1983. WESENBERG, B. Beiträge zur Rekonstruktion griechischer Architektur nach literarischen Quellen. Berlin, Mann.

1984. KNELL, H.; WESENBERG, B. Vitruv-Kolloquium des Deutschen Archäologen-Verbandes e.V., durchgefuhrt an der Technischen Hochschule Darmstadt, 17. bis 18. Juni 1982. Darmstadt, Technische Hochschule. 
1985. KNELL, H. Vitruvs Architekturtheorie: Versuch einer Interpretation. Darmstadt, Wissenschaftliche Buchgesellschaft.

1989. GEERTMAN, H.; DE JONG, J. J. (eds.) Munus non ingratum: proceedings of the International Symposium on Vitruvius' De Architectura and the Hellenistic and Republican Architecture, Leiden, 2023 January 1987. Leiden, Stichting Bulletin antieke beschaving.

1994. Le projet de Vitruve: objet, destinataires et réception du De architectura: actes du colloque international organisé par l'Ecole française de Rome, l'Institut de recherche sur l'architecture antique du CNRS et la Scuola normale superiore de Pise (Rome, 26-27 mars 1993). Roma, École française de Rome.

1997. ROLF, R. Vitruviuscongres: Heerlen, 13, 14, 15 oktober 1995; Maastricht, 24 oktober 1995. Heerlen, Vitruvianum.

\section{VITRÚVIO: ARTIGOS SOBRE ESTUDOS VITRUVIANOS PARA OS TEMPLOS GREGOS}

1952 (1953). RIEMANN, H. Vitruv und der griechische Tempel. AA, p. 138.

1953. RIEMANN, H. Review: [untitled]: MOE, C. J. Numeri di Vitruvio. Milano, Edizioni del Milione, 1945. Gnomon, 25, p. 248-250.

1974. PANNUTI, U. Il modulo nell'architettura dórica. RendLinc, 29, p. 431-452.

1977. KURENT, T.; MUHIČ, L. Vitruvius on Module. Archeoloski Vestnik. Academia Scientarum et artium Slovenica, 28, p. 209-232.

1979. FALUS, R. Sur la Théorie de Module de Vitruve. AantHung, 31, p. 249-270.

1984. KNELL, H. Die Hermogenes-Anedokte und das Endedes dorischen Ringhallentempels. In: KNELL, H.; WESENBERG, B. (Hrsg.), VitruvKolloquium des Deutschen Archä̈logen-Verbandes e.V., durchgefuhrt an der Technischen Hochschule Darmstadt, 17. bis 18. Juni 1982. Darmstadt, Technische Hochschule, p. 41-64.

1984. WESENBERG, B. Vitruvs griechischer Tempel. In: KNELL, H.; WESENBERG, B. (Hrsg.), Vitruv-Kolloquium des Deutschen Archäologen-Verbandes e.V., durchgefuhrt an der Technischen 
Hochschule Darmstadt, 17. bis 18. Juni 1982. Darmstadt, Technische Hochschule, p. 65-96.

1994. WESENBERG, B. Die Bedeutung des Modulus in der Vitruvianischen Tempelarchitektur. In: Le projet de Vitruve: objet, destinataires et réception $\mathrm{du}$ De architectura: actes du colloque international organisé par l'Ecole française de Rome, l'Institut de recherche sur l'architecture antique du CNRS et la Scuola normale superiore de Pise (Rome, 26-27 mars 1993). Roma, École française de Rome, p. 90-104.

1989. COULTON, J. J. Modules and Measurements in Ancient Design and Modern Scholarship. In: GEERTMAN, H.; DE JONG, J. J. (eds.) Munus non ingratum: proceedings of the International Symposium on Vitruvius' De Architectura and the Hellenistic and Republican Architecture, Leiden, 2023 January 1987. Leiden, Stichting Bulletin antieke beschaving, p. 85-89. 